\begin{tabular}{|c|l|}
\hline Title & Improvement of Potential Drop Predictions for Plasma Wind Tunnels by Cathode Sheath \\
\hline Author(s) & Takahashi, Y usuke; Kihara, Hisashi; A be, Ken-ichi \\
\hline Citation & $\begin{array}{l}\text { Journal of Thermophysics and Heat Transfer, 26(3), 540-544 } \\
\text { https://doi.org/L0.2514/L.T3723 }\end{array}$ \\
\hline Issue Date & 2012-07 \\
\hline Doc URL & http://hdl.handle.net/2115/56574 \\
\hline Rights & ○2012 A merican Institute of A eronautics and A stronautics \\
\hline Type & article (author version) \\
\hline File Information & Improvement of Potential Drop Predictions for Plasma Wind Tunnels by Cathode Sheath.pdf \\
\hline
\end{tabular}

Instructions for use 


\title{
Improvement of Potential Drop Predictions for Plasma Wind Tunnels by Cathode Sheath
}

\author{
Yusuke Takahashi, ${ }^{1}$ Hisashi Kihara ${ }^{2}$ and Ken-ichi Abe $^{3}$ \\ Kyushu University, 744 Motooka, Nishi-ku, Fukuoka 819-0395, Japan
}

\section{Nomenclature}

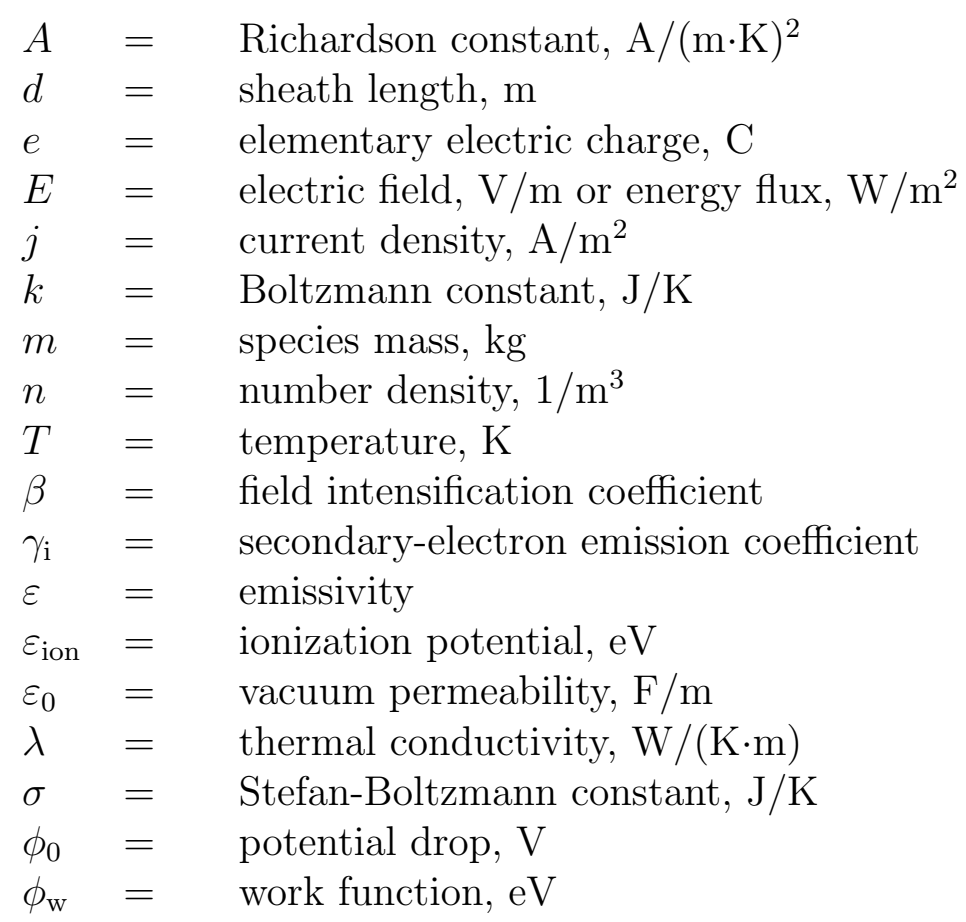

Subscripts

$\begin{array}{lll}\mathrm{amb} & = & \text { ambipolar flux } \\ \mathrm{d} & = & \text { sheath edge } \\ \mathrm{e} & = & \text { electron } \\ \mathrm{ele} & = & \text { electron flux } \\ \mathrm{fe} & = & \text { field-electron emission } \\ \mathrm{i} & = & \text { ion } \\ \mathrm{rad} & = & \text { radiation } \\ \mathrm{se} & = & \text { secondary-electron emission } \\ \mathrm{te} & = & \text { thermionic-electron emission } \\ \text { tot } & = & \text { total flux } \\ \mathrm{w} & = & \text { wall }\end{array}$

\footnotetext{
${ }^{1}$ JSPS Research Fellow, Department of Aeronautics and Astronautics; (Present address: Japan Aerospace Exploration Agency, 3-1-1 Yoshinodai, Chuo-ku Sagamihara, Kanagawa 252-5210, Japan; yusuketakahashi@gd.isas.jaxa.jp.)

${ }^{2}$ Assistant Professor, Department of Aeronautics and Astronautics.

${ }^{3}$ Professor, Department of Aeronautics and Astronautics.
} 


\section{Introduction}

An arc-heated wind tunnel is very useful for ground-based experiments of the planet-entry environment. Its applications include performance tests of thermal protection systems. To perform such experiments with high accuracy, it is essential to understand the physical properties of the free jets discharged from the nozzle. However, the flow field in an arc heater is very complicated due to the complex phenomena associated with the arc discharge in the constrictor as well as the strong thermochemical nonequilibrium in the nozzle. Consequently, it is extremely difficult to simultaneously measure all the properties of the free jets. On the other hand, due to the development of high-performance computers, numerical simulation has become a powerful tool for investigating the details of complex flow fields.

So far, several research groups [1-3] have investigated arc-heated flow fields by computational fluid dynamics (CFD). To achieve accurate predictions, it is indispensable to properly model the heating process in the constrictor section and the expansion phenomena in the nozzle section. In our previous works [4-6], numerical simulations were performed for various arc-heated flows. Included are the Kyushu University $20 \mathrm{~kW}$ arc-heated wind tunnel (KUWT) [7], the JAXA $750 \mathrm{~kW}$ arc-heated wind tunnel (JXWT) [8] and the NASA 20 MW Aerodynamic Heating Facility (AHF) [9]. Note that the first one is categorized as a constrictor-type arc heater $(\mathrm{CAH})$ and the last two are categorized as segmented-type arc heaters (SAH). As is summarized in Refs. $[10,11]$, the cathode configuration of the former is rod-shaped, while that of the latter is ring-shaped.

Considering the computational results of the mass-averaged enthalpy, the Pitot pressure and the heat flux, we expect that our analysis models properly predicted the net energy input into the flow fields in the facilities. On the other hand, however, the calculated arc voltage was considerably underestimated for all the cases. As reported in Refs. [5,6], the predicted voltages are about 15 volt for $\mathrm{CAH}$ and several hundred volt for SAH lower than the measured ones. Moreover, for SAH, the test gas was assumed to be air in these references, while an air-argon mix model is expected to be more realistic. However, an argon-gas mixture may cause further underprediction of the arc voltage as reported in Ref. [12]. We believe that these discrepancies between the computed and measured arc voltages are caused mainly by the potential drop in sheath.

The sheath is formed near the wall surface of an electrode in an arc-heating facility due to the difference between the mobility of ions and electrons. The electrons with high mobility immediately reach the wall and then create a negative wall potential (a drop in the electric potential). In the sheath envelop between the plasma region and the wall surface, there are steep gradients of physical properties, such as electron temperature, electric potential and number density. In general, the thickness of a sheath is on the order of micrometers and roughly corresponds to the order of the Debye length [13]. Therefore, the sheath is very thin and the physical properties there are expected to be distributed one-dimensionally.

When we operate an arc-heating facility, the arc voltage measured may include the potential drop generated by sheath on the electrodes. It is thought that the potential drop never contributes to the energy input into the flow field and just results in energy loss. Therefore, in order to predict the performance of an arc-heated wind tunnel more accurately, it is important to investigate sheath phenomena. Needless to say, such a high-accuracy prediction technique is definitely useful for designing a new arc-heating facility in the future.

As for the cathode material in $\mathrm{CAH}$, two-percent thoriated tungsten is commonly used. Hot cathode materials such as tungsten and graphite have a higher melting point. Moreover, thoriated tungsten is a good emitter because of its lower work function. When the wall 
temperature becomes very high in the discharge region, the thermionic-electron emission is one of the dominant mechanisms to emit electrons from the wall surface. So far, sheath behaviors for $\mathrm{CAH}$, an arcjet thruster and a discharge lamp, which focus on the thermionic mechanism, have been investigated by some research groups [14-16]. On the other hand, there are few studies for SAH, in which copper is used for the cathode material. Since cold cathode materials such as copper have lower values for both melting and boiling points, their maximum allowable temperature is relatively low such that thermionic emission is too small to maintain a discharge in the plasma. In this case, it is thought that the field-electron emission becomes dominant. This emission mechanism may cause a large potential drop near the cathode and thus it greatly influences the prediction accuracy of CFD. To increase the reliability of the sheath models introduced into a CFD code, it is desirable that the developed model is applicable to various types of arc heaters such as CAH and SAH cases. Hence, further detailed study is required for the discharge mechanism in both $\mathrm{CAH}$ and SAH cases.

The main objective of the present study is to clarify the potential drop near the electrode surface, in order to calculate the arc voltage for various arc heaters more accurately. We focus particularly on the sheath phenomena near the cathode wall surface. To discharge the arc current, large amounts of electrons need to be generated near the cathode surface, for which high temperature on the wall surface and/or strong electric field in sheath are necessary. On the other hand, these factors are not needed for absorption of electrons near the anode surface because electrons in the discharge region easily drift toward the anode surface. It is thus thought that the potential drop on the anode is relatively small compared with that on the cathode. To investigate these phenomena in detail, we consider two representative arc-heating facilities (i.e., $\mathrm{CAH}$ and $\mathrm{SAH}$ ).

\section{Physical and Mathematical Models for Sheath}

\subsection{Electron-Emission Models}

In the present study, for the electron-generation mechanisms near the cathode surface, we consider the electron $\left(j_{\text {ele }}\right)$ and ion $\left(j_{\text {amb }}\right)$ fluxes drifting toward the cathode wall from the plasma region. On the other hand, from the cathode wall to the plasma region, the thermionicelectron $\left(j_{\text {te }}\right)$, secondary $\left(j_{\mathrm{se}}\right)$ and field $\left(j_{\mathrm{fe}}\right)$ emissions are taken into account. Note that it is assumed that no collisions between particles occurs in the sheath layer and the electron flux generated by ionization reactions can be neglected in the present model. These electron/ion fluxes are balanced in the sheath layer (current continuity). The flux balance is expressed as follows:

$$
j_{\mathrm{tot}}=-j_{\mathrm{ele}}-j_{\mathrm{amb}}+j_{\mathrm{te}}+j_{\mathrm{se}}+j_{\mathrm{fe}},
$$

where $j_{\text {tot }}$ represents the total current density in the plasma. Each current density is described in detail later. Equation (1) is numerically solved with respect to the potential drop $\left(\phi_{0}\right)$ by the Newton-Raphson method.

The electric field $(E)$ is obtained by solving the following Poisson's equation:

$$
\varepsilon_{0} \frac{d^{2} \phi(x)}{d x^{2}}=e\left\{n_{\mathrm{e}}(x)-n_{\mathrm{i}}(x)\right\} .
$$

Distribution of the number density of electrons in sheath is given by the Boltzmann relation 
as,

$$
n_{\mathrm{e}}(x)=n_{\mathrm{ed}} \exp \left(\frac{e \phi(x)}{k T_{\mathrm{e}}}\right) .
$$

Then, the equation for the number density of ions is derived from the equation of continuity and the energy conservations of ions in sheath as follows:

$$
n_{\mathrm{i}}(x)=\frac{n_{\mathrm{id}}}{\sqrt{1-\frac{2 e \phi(x)}{k T_{\mathrm{e}}}}} .
$$

Note that the number densities of ions and electrons are assumed to be equal at the sheath edge $\left(n_{\mathrm{ed}}=n_{\mathrm{id}}\right)$. Equations (2), (3) and (4) are analytically solved with regard to the potential drop, and the distributions of the electric field and the number densities in sheath are obtained.

\subsubsection{Electron and ion fluxes}

When electrons drift to the sheath layer, only those electrons having energy higher than the potential barrier of the sheath can reach the cathode wall surface. Such an electron flux is given by

$$
j_{\text {ele }}=e n_{\mathrm{ed}} \sqrt{\frac{k T_{\mathrm{e}}}{2 \pi m_{\mathrm{e}}}} \exp \left(\frac{e \phi_{0}}{k T_{\mathrm{e}}}\right) .
$$

The ion flux at the sheath edge corresponds to the ambipolar current drifting from the plasma, which is given by

$$
j_{\mathrm{amb}}=-e n_{\mathrm{id}} \sqrt{\frac{k T_{\mathrm{e}}}{m_{\mathrm{i}}}} .
$$

As described in Eq. (6), the ion velocity is given by the Bohm velocity at the sheath edge.

\subsubsection{Thermionic-electron emission}

Electrons can be emitted from the wall surface due to high temperature of the cathode. The energy difference between the vacuum and Fermi levels for a given material is defined as the local work function. Thus, if the temperature is high enough, thermionic electron is easily emitted from the material surface. Moreover, the electric field reduces both the Fermi level and the work function and thus increases the emission of electrons, a process which is referred to as the Schottky effect. The thermionic-electron emission is expressed by the Richardson-Dushman equation with the Schottky effect as follows:

$$
j_{\mathrm{te}}=A T_{\mathrm{w}}^{2} \exp \left\{-\frac{e\left(\phi_{\mathrm{w}}-\phi_{\mathrm{E}}\right)}{k T_{\mathrm{w}}}\right\},
$$

where

$$
\phi_{\mathrm{E}}=\sqrt{\frac{e E}{4 \pi \varepsilon_{0}}} .
$$

The Richardson constant $(A)$ and the work function $\left(\phi_{\mathrm{w}}\right)$ for thoriated tungsten are respectively set to $3.0 \times 10^{4} \mathrm{~A} /(\mathrm{m} \cdot \mathrm{K})^{2}$ and $2.63 \mathrm{eV}$, while those for copper are $1.2 \times 10^{6} \mathrm{~A} /(\mathrm{m} \cdot \mathrm{K})^{2}$ and $4.41 \mathrm{eV}$, respectively. 


\subsubsection{Secondary-electron emission}

When an ionic species collides with the wall, electrons are emitted from the wall surface, if the collision energy exceeds the work function of the wall material. The current density of this secondary emission by ion species is given with the ambipolar current as follows:

$$
j_{\mathrm{se}}=-\gamma_{\mathrm{i}} j_{\mathrm{amb}},
$$

Although the secondary-electron emission coefficient $\gamma_{\mathrm{i}}$ changes according to incident angle and energy, it is reported that the coefficient is almost constant for ion energies below several hundred electron volt and its value is approximately 0.1 [17]. Therefore, in this study, the secondary-electron emission coefficient $\gamma_{\mathrm{i}}$ is set to 0.1 .

\subsubsection{Field-electron emission}

When there exist strong electric fields, electrons are emitted from the wall material by quantum tunneling. Current density of the field emission can be obtained by the Fowler-Nordheim equation given by

$$
j_{\mathrm{fe}}=\frac{1.54 \times 10^{-2}(\beta E)^{2}}{\phi_{\mathrm{w}}} \exp \left\{-\frac{6.83 \times 10^{9} \phi_{\mathrm{w}}^{3 / 2} v(y)}{\beta E}\right\} .
$$

The field intensification factor $\beta$, which is a parameter to express local enhancement of the field by small projections on electrode surface, is set to be 150 , following the previous studies $[18,19]$. According to Ref. [20], the functions $y$ and $v(y)$ are obtained by

$$
\begin{gathered}
y=3.79 \times 10^{-5} \frac{\sqrt{\beta E}}{\phi_{\mathrm{w}}}, \\
v(y)=(1+\eta)^{-\frac{3}{2}}\left\{\left(a_{0}+a_{1} \eta+\cdots+a_{5} \eta^{5}\right)+\left(b_{1} \eta+\cdots+b_{5} \eta^{5}\right) \ln \left(\frac{1}{\eta}\right)\right\},
\end{gathered}
$$

where the constants for the Fowler-Nordheim function are summarized in Table 1 and $\eta$ is given by

$$
\eta=\frac{1-\sqrt{1-y^{2}}}{1+\sqrt{1-y^{2}}}
$$

\section{$2.2 \quad$ Energy Fluxes}

In the sheath layer, the energy-conservation equation is derived from the balance of the energy fluxes at the cathode surface. In this study, it is assumed that electrons and ions reaching the cathode surface are perfectly absorbed at the surface. Note that erosion and evaporation of the cathode material are neglected. We consider the energy fluxes transported by the electrons $\left(E_{\text {ele }}\right)$ and the ions $\left(E_{\mathrm{amb}}\right)$ from the plasma region, the thermionic-electron $\left(E_{\mathrm{te}}\right)$, secondary $\left(E_{\mathrm{se}}\right)$ and field $\left(E_{\mathrm{fe}}\right)$ emissions, and the energy fluxes cooling the cathode surface by the electron emission. Moreover, the radiation power $\left(E_{\mathrm{rad}}\right)$ emitted from the cathode surface, the convective heat transfer $\left(q_{\mathrm{e}}\right)$ into the cathode across the sheath and the heat conduction $\left(q_{\text {cond }}\right)$ into the cathode are introduced. Thus, the energy balance is obtained by

$$
q_{\mathrm{e}}=E_{\mathrm{ele}}+E_{\mathrm{amb}}+E_{\mathrm{te}}+E_{\mathrm{se}}+E_{\mathrm{fe}}+E_{\mathrm{rad}}+q_{\mathrm{cond}} .
$$


which is expressed in detail as

$$
\begin{aligned}
-\lambda \frac{\partial T_{\mathrm{e}}}{\partial r} & =j_{\text {ele }}\left(\phi_{\mathrm{w}}+\frac{2 k}{e} T_{\mathrm{e}}\right)-j_{\mathrm{amb}}\left(-\phi_{0}+\varepsilon_{\text {ion }}-\phi_{\mathrm{w}}+\frac{2 k}{e} T_{\mathrm{w}}\right) \\
& -\left(j_{\mathrm{te}}+j_{\mathrm{se}}+j_{\mathrm{fe}}\right)\left(\phi_{\mathrm{w}}+\frac{2 k}{e} T_{\mathrm{w}}\right)-\varepsilon \sigma T_{\mathrm{w}}^{4}+q_{\mathrm{cond}}
\end{aligned}
$$

where $r$ is the direction orthogonal from the cathode surface to the plasma region and $\varepsilon_{\text {ion }}$ is the ionization potential of the test gas. The emissivity $\varepsilon$ is set to be 0.3 for tungsten $(\mathrm{CAH})$ and 0.1 for copper (SAH) [21], respectively.

\section{Results and Discussion}

The present test cases are constrictor-type $(\mathrm{CAH})$ and the segmented-type (SAH) arc heaters. In general, the cathode material of CAH is thoriated tungsten and that for SAH is copper. The former is referred to as the hot-cathode material and the latter is referred to as a coldcathode material. Note that the cathode-surface temperature is kept below the melting point in all the calculations. As reported in Refs. $[5,6]$, the total and ambipolar current densities for $\mathrm{CAH}$ are much larger than those for SAH. Such a high current density is a common feature that is seen in $\mathrm{CAH}$ because the current is highly concentrated in the vicinity of the cathode. In the present study, the baseline cases are set to $j_{\text {tot }}=-10^{9}, j_{\mathrm{amb}}=-10^{6}$ and $q_{\mathrm{cond}}=-10^{7}$ for CAH and $j_{\text {tot }}=-10^{7}, j_{\mathrm{amb}}=-10^{6}$ and $q_{\mathrm{cond}}=-10^{7}$ for $\mathrm{SAH}$, respectively.

Figure 1 shows the profiles of the potential drop and the electron temperature versus the ambipolar current for three conditions of total current. The wall temperatures are simply set to be $3600 \mathrm{~K}$ for $\mathrm{CAH}$ and $1000 \mathrm{~K}$ for $\mathrm{SAH}$, respectively. Although these temperatures do not always correspond to the max allowable temperature of the cathode material, the actual values are possibly lower due to the water-cooling device for the cathode. As seen in Fig. 1(a), difference of arc voltage for $\mathrm{CAH}$ predicted by the sheath model is only several dozen volt. On the other hand, concerning SAH, it is found from Fig. 1(b) that the sheath model causes much larger difference in voltage and it becomes several hundred volt. Although the scale of SAH (e.g., JXWT) is generally much larger than that of CAH (e.g., KUWT), there may be another reason based on the heating mechanism in each facility. To clarify this, we investigate the characteristics of the phenomena in more detail.

Figure 2 shows the potential drops and the current densities versus the wall temperature for the baseline case. In the figure, some remarkable features can be seen. In the lower temperature region, the field-emission current $\left(j_{\mathrm{fe}}\right)$ is dominant, while the thermionic-emission current $\left(j_{\text {te }}\right)$ rapidly increases with decrease of $j_{\text {fe }}$ as the wall temperature becomes high. The CAH facility uses a hot-cathode material (i.e., thoriated tungsten) whose melting point is around $T_{\mathrm{w}} \sim 3600 \mathrm{~K}$. As seen in Fig. $2(\mathrm{a})$, the thermionic-emission current $\left(j_{\text {te }}\right)$ is comparable with the field-emission current in this wall-temperature range. On the other hand, the coldcathode material (i.e., copper) is used in the SAH facility, where the melting point is around $T_{\mathrm{w}} \sim 1000 \mathrm{~K}$. In this lower-temperature region, the field-emission current $\left(j_{\mathrm{fe}}\right)$ is dominant, as seen in Fig. 2(b). Secondary emission $\left(j_{\text {se }}\right)$ is always relatively low for all the cases. Although it is difficult to determine an accurate value of the secondary emission coefficient $\left(\gamma_{i}\right)$ in Eq. (9), the ambipolar current is relatively low in this study and thus it is thought that the uncertainty for this coefficient has a minor influence on the predictive accuracy of the potential drop. On the other hand, the sensitivity of the potential drop to the field intensification factor $(\beta)$ is large in the model, while it is in fact very difficult to determine 
its accurate value. It is expected that the value of $\beta$ used in this study is reasonable, more detailed discussion may be needed in the future.

Profiles of the potential drop, the electric field and the number densities of ions and electrons in the sheath layer are shown in Fig. 3. The sheath length seems to be of the order of micrometers and very thin compared with the characteristic length of the cathode length which is typically of the order of centimeters. Since the magnitude of the potential drop at the cathode surface is small in CAH making the electric field weak, more electrons can remain in the sheath. Conversely, the sheath layer is almost filled by ions and most electrons return to the plasma in the sheath for $\mathrm{SAH}$, because the potential drop results in being large. In the model, if the number density of ions at the sheath edge (i.e., the ambipolar current) decreases, the strength of the electric field strength also decreases. This causes the decrease of the field emission $\left(j_{\mathrm{fe}}\right)$ and, at the same time, the magnitude of the potential drop must increase to maintain discharge. As seen in Fig. 1(b), the increase of magnitude of the potential drop with the decrease of the field emission corresponds to the condition below $\left|j_{\mathrm{amb}}\right|=10^{5}$. In the CAH case shown in Fig. 1(a), if the ambipolar current becomes small, the magnitude of the potential drop moderately decreases or hardly occurs. This is because the electron-emission mechanism for $\mathrm{CAH}$ is mainly due to both the thermionic and field emissions. Such being the case, there can be seen some notable differences between CAH and SAH. This is thought to be caused by the difference of the cathode feature between these facilities, i.e., the cathode material and the max allowable wall temperature.

\section{Conclusions}

In order to investigate the potential drop due to the sheath for arc-heated wind tunnels, a simple cathode-sheath model was developed. The present model was applied to two representative different types of arc heaters i.e., the constrictor-type arc heater $(\mathrm{CAH})$ and the segmented-type arc heaters (SAH). By using the computational results, we tried to investigate the phenomena occurring in the sheath layer. As for $\mathrm{CAH}$, the predicted potential drops were possibly several dozen volt. In this case, the cathode-surface temperature is very high and then the thermionic emission plays an important role to emit electrons from such a hot-cathode material (e.g., tungsten). On the other hand, several hundred volts of potential drop was obtained for SAH. The wall temperature was relatively low at the cathode and thus the field emission becomes dominant for such a cold-cathode material (e.g., copper). From these results, we consider the present sheath model to have the fundamental capability of reasonably explaining the potential drop appearing in various kinds of arc-heating facilities, though there still remains some points to be improved in the future.

\section{Acknowledgments}

The first author is supported by Research Fellowships of the Japan Society for the Promotion of Science for Young Scientists. The computation was mainly carried out using the computer facilities at Research Institute for Information Technology, Kyushu University. 


\section{References}

[1] C. Park and S.-H. Lee. "Validation of Multitemperature Nozzle Flow Code". Journal of Thermophysics and Heat Transfer, Vol. 9, No. 1, pp. 9-16, Jan.-Mar. 1995.

[2] T. Sakai. "Computational Simulation of High-Enthalpy Arc Heater Flows". Journal of Thermophysics and Heat Transfer, Vol. 21, No. 1, pp. 77-85, Jan.-Mar. 2007.

[3] J.-P. Lee, C. Kim, and K.-H. Kim. "Accurate Computations of Arc-Heater Flows Using Two-Equation Turbulence Models". Journal of Thermophysics and Heat Transfer, Vol. 21, No. 1, pp. 67-76, Jan.-Mar. 2007.

[4] Y. Takahashi, H. Kihara, and K. Abe. "Numerical Investigation of Nonequilibrium Plasma Flows in Constrictor- and Segmented-Type Arc Heaters". Journal of Thermophysics and Heat Transfer, Vol. 24, No. 1, pp. 31-39, 2010.

[5] Y. Takahashi, H. Kihara, and K. Abe. "The Effects of Radiative Heat Transfer in Arc-Heated Nonequilibrium Flow Simulation". Journal of Physics D: Applied Physics, Vol. 43, No. 18, p. 185201, 2010.

[6] Y. Takahashi, H. Kihara, and K. Abe. "Turbulence and Radiation Behaviours in LargeScale Arc Heaters". Journal of Physics D: Applied Physics, Vol. 44, No. 8, p. 085203, 2011.

[7] M. Hirakawa, K. Abe, M. Nishida, K. Takeishi, and M. Matsuura. "Application of a 20 kW Arc-Heated Wind Tunnel to Evaluation Tests of Wall Catalysis". Transactions of the Japan Society for Aeronautical and Space Sciences, Vol. 45, No. 150, pp. 217-223, 2003.

[8] T. Matsuzaki, K. Ishida, Y. Watanabe, K. Miho, H. Itagaki, and T. Yoshinaka. "Constructions and Characteristics of the $750 \mathrm{~kW}$ Arc Heated Wind Tunnel". NAL TM-760, National Aerospace Laboratory, Chofu, Japan, 2001.

[9] T. M. Hightower, J. A. Balboni, C. L. M. Donald, K. F. Anderson, and E. R. Martinez. "Enthalpy by Energy Balance for Aerodynamic Heating Facility at NASA Ames Research Center Arc Jet Complex". In Proceedings of the 48th International Instrumentation Symposium, ISA TP02-AERO-2000, Instrumentation, Systems, and Automation Society, San Diego, CA, USA, 2002.

[10] M. A. Birkan. "Arcjets and Arc Heaters: An Overview of Research Status and Needs". Journal of Propulsion and Power, Vol. 12, No. 6, pp. 1011-1017, Nov.-Dec. 1996.

[11] M. Hinada, Y. Inatani, T. Yamada, and K. Hiraki. "An Arc-Heated High Enthalpy Test Facility for Thermal Protection Studies". ISAS Report 664, Institute of Space and Aeronautics Science, Sagamihara, Japan, March 1996.

[12] J.-P. Lee, S.-H. Han, C. Kim, and K.-H. Kim. "Analysis of Segmented Arc-Heated Flows with High Argon Concentration". Journal of Thermophysics and Heat Transfer, Vol. 22, No. 2, pp. 187-200, April-June 2008.

[13] M. S. Benilov. "Analysis of ionization non-equilibrium in the near-cathode region of atmospheric-pressure arcs". Journal of Physics D: Applied Physics, Vol. 32, pp. 257262, 1999. 
[14] J. J. Lowke, R. Morrow, and J. Haidar. "A Simplified Unified Theory of Arcs and Their Electrodes". Journal of Physics D: Applied Physics, Vol. 30, No. 14, pp. 2033-2042, 1997.

[15] J. Lu, H. Krier, R. L. Burton, and K. D. Goodfellow. "Cathode Sheath Voltage Models for Hydrazine Arcjets". Journal of Thermophysics and Heat Transfers, Vol. 12, No. 2, pp. 230-238, April-June 1998.

[16] K. Fujita and Y. Arakawa. "Performance Computation of a Low-Power Hydrogen Arcjet". Journal of Propulsion and Power, Vol. 15, No. 1, pp. 144-150, Jan.-Feb. 1999.

[17] A. V. Phelps and Z. L. Petrovic. "Cold-cathode discharges and breakdown in argon: surface and gas phase production of secondary electrons". Plasma Sources Science and Technology, Vol. 8, No. 3, pp. R21-R44, 1999.

[18] H. Tomaschke and D. Alpert. "Field Emission from a Multiplicity of Emitters on a Broad-Area Cathode". Journal of Applied Physics, Vol. 38, No. 2, pp. 881-883, 1967.

[19] G. A. Farrall. "Numerical Analysis of Field Emission and Thermally Enhanced Emission from Broad-Area Electrodes in Vacuum". Journal of Applied Physics, Vol. 41, No. 2, pp. 563-571, 1970.

[20] H. C. Miller. "Values of Fowler-Nordheim Field Emission Functions: v(y), t(y), and s(y)". Journal of The Franklin Institute, Vol. 282, pp. 382-388, 1966.

[21] E. A. Brandes. Smithells Metals Reference Book: Sixth Edition. Butterworths, London, 1983.

Table 1: Constants for Fowler-Nordheim function

\begin{tabular}{cc|cc}
\hline \hline$a_{0}$ & 1.0 & & \\
$a_{1}$ & -1.329337308 & $b_{1}$ & -0.750016317 \\
$a_{2}$ & 0.312530542 & $b_{2}$ & 0.092013612 \\
$a_{3}$ & 0.038378000 & $b_{3}$ & -0.004906196 \\
$a_{4}$ & -0.009912374 & $b_{4}$ & -0.020665635 \\
$a_{5}$ & -0.011658860 & $b_{5}$ & -0.003571244 \\
\hline \hline
\end{tabular}




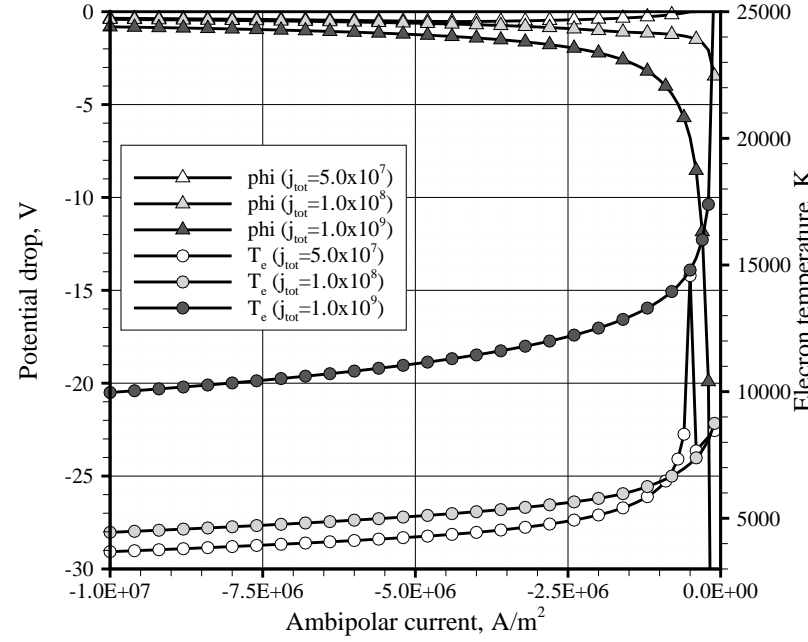

(a) Constrictor-type arc heater

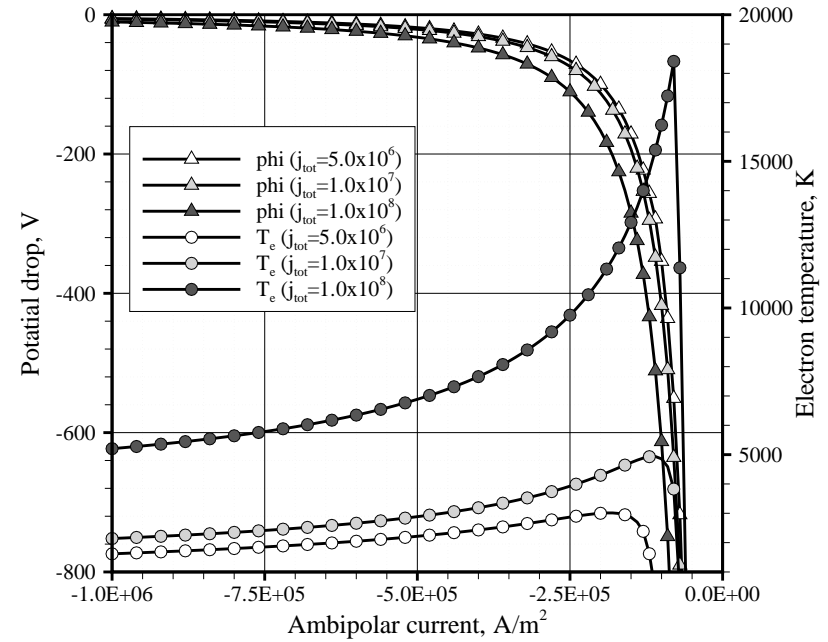

(b) Segmented-type arc heater

Figure 1: Profiles of potential drop and electron temperature versus ambipolar current

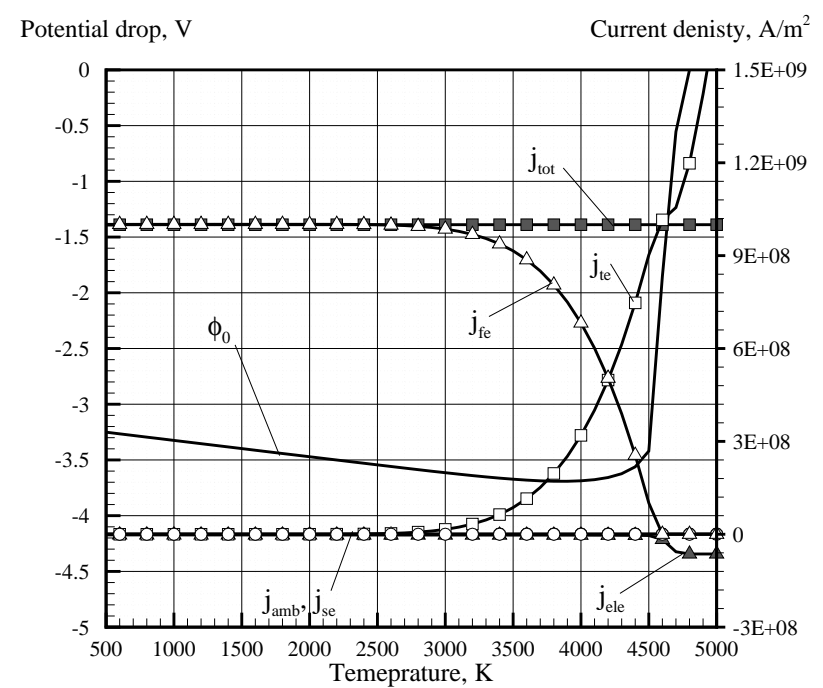

(a) Constrictor-type arc heater

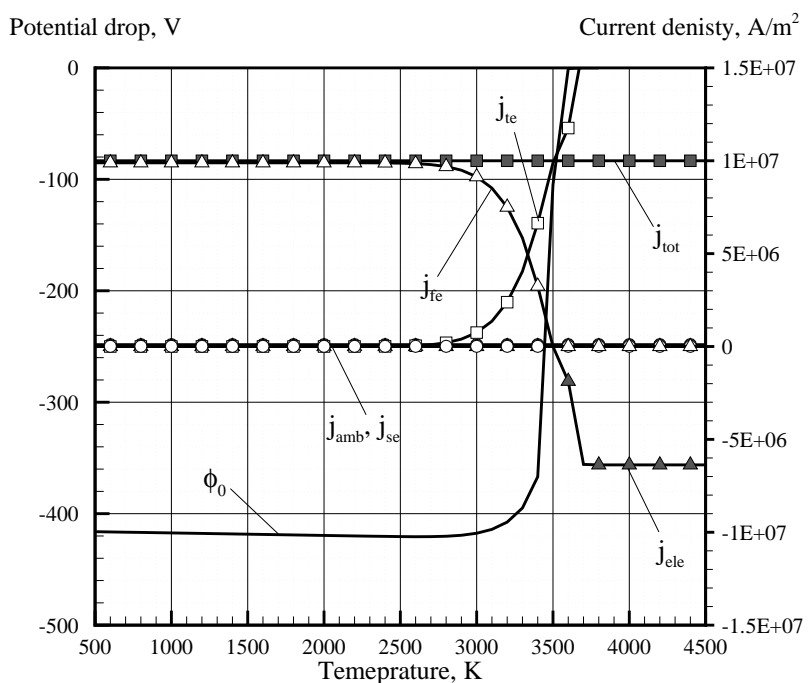

(b) Segmented-type arc heater

Figure 2: Profiles of potential drop and current densities versus wall temperature 


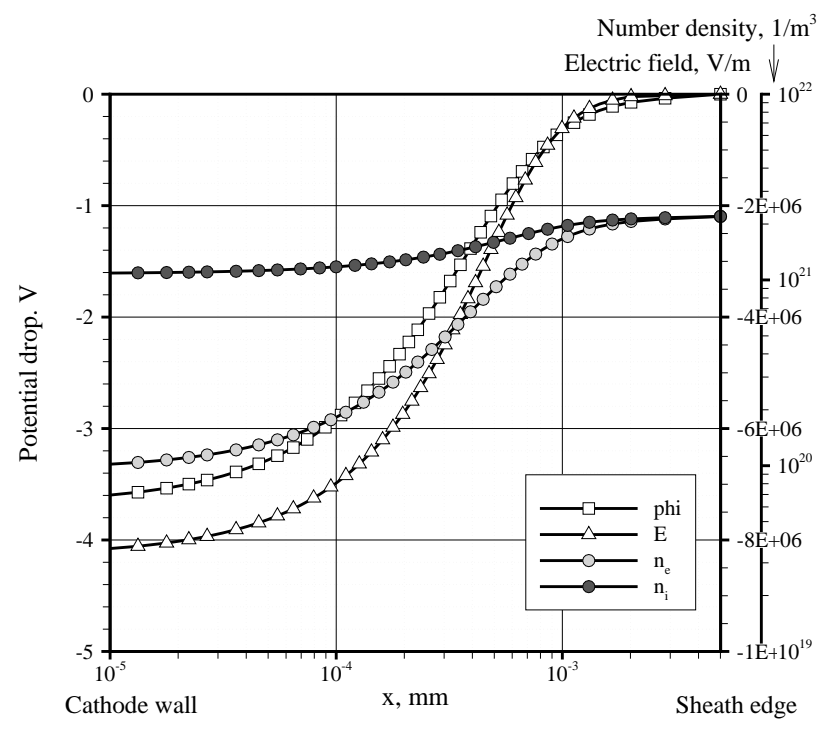

(a) Constrictor-type arc heater

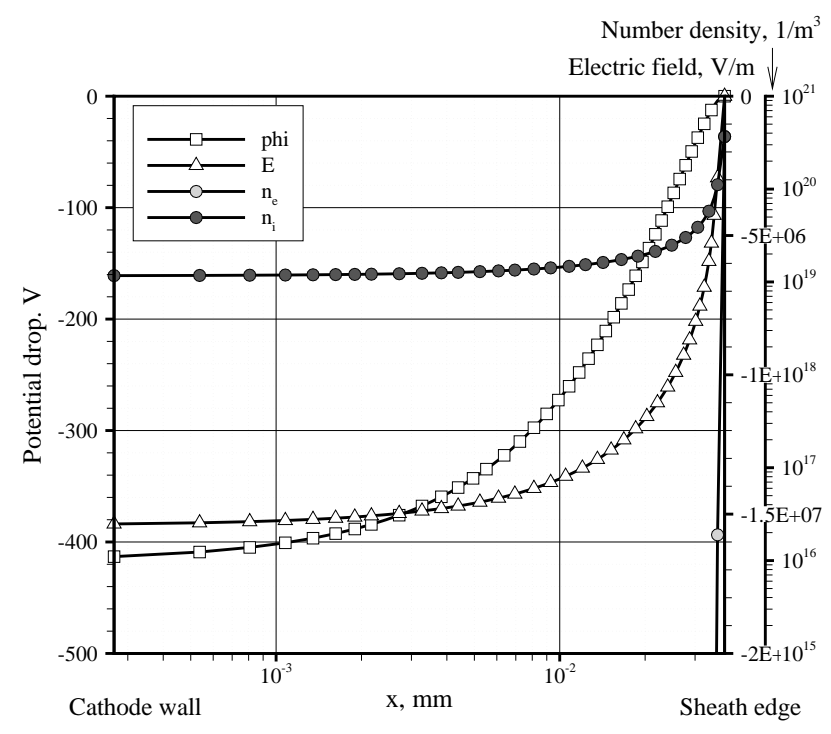

(b) Segmented-type arc heater

Figure 3: Profiles of the potential drop, electric field and number densities of ions and electrons in sheath layer 